\title{
Experimental study on the regulation of erlotinib-induced radiosensitization with an anti-c-MET monoclonal antibody
}

Hong-Qing Zhuang ${ }^{1,2^{*}+}$, Hongxia Zhuang ${ }^{3 \dagger}$, Qifu Bo ${ }^{4 \dagger}$, Yihang Guo ${ }^{1,2 \dagger}$, Jun Wang ${ }^{1,2}$, Lu-Jun Zhao ${ }^{1,2}$, Zhi-Yong Yuan ${ }^{1,2}$ and Ping Wang ${ }^{1,2}$

\begin{abstract}
Purpose: Erlotinib is a novel therapeutic agent for cancer treatment. This study was performed to investigate the role of c-MET-PI3K-AKT pathway in the regulation of erlotinib-induced radiosensitization.

Methods: A973 lung adenocarcinoma cells treated with 6 Gy of radiation were incubated in the presence of erlotinib. The apoptotic rate after 24 hours, the colony-formating rate after 14 days, and changes in the c-MET expression levels after 14 days of irradiation were examined. Surviving fractions in different treatment groups (blank control, radiation alone, erlotinib alone, anti-c-MET monoclonal antibody alone, combined erlotinib and radiation, and combined erlotinib and radiation with anti-c-MET monoclonal antibody groups) were determined, the survival curves were plotted, and the sensitizer enhancement ratio was calculated using colony formation assays. Expressions of c-MET, p-C-MET, PI3K, AKT, and p-AKT in cells in different treatment groups were examined by Western blot analysis.

Results: The apoptotic rate in the combined erlotinib and radiation group was higher than those in single treatment groups; however, the colony-forming rate remained approximately $2.04 \pm 1.02 \%$. The expression of c-MET in colony-forming cells in the combined group significantly increased, and the blockade of c-MET activity significantly enhanced the radiosensitizing effect of erlotinib. The expression of c-Met, P-c-MET, PI3K, AKT, and p-AKT among colony-forming cells significantly decreased upon the inhibition of c-MET.

Conclusions: Upregulated activity of the c-MET-PI3K-AKT pathway was found to be important for cell survival under combined the treatment with erlotinib and radiation. The blockade of the c-MET-PI3K-AKT signaling pathway enhanced the radiosensitizing effect of erlotinib.
\end{abstract}

Keywords: Tyrosine kinase inhibitor, Radiosensitization, Radiation resistance, Acquired drug resistance, PI3K pathway

\section{Introduction}

A number of previous studies have demonstrated a clear radiosensitizing effect of erlotinib treatment [1-3]. However, the combined treatment with erlotinib and radiation sometimes exhibits poor antitumor effects [4]. Our previous report suggested that the extent of antitumor effects of the combined erlotinib and radiation treatment may be related to the PI3K pathway [5], although

\footnotetext{
* Correspondence: hongqingzhuang@163.com

${ }^{\dagger}$ Equal contributors

${ }^{1}$ Department of Radiotherapy, Tianjin Medical University Cancer Institute and Hospital, National Clinical Research Center for Cancer, Tianjin Key Laboratory of Cancer Prevention and Therapy, Tianjin, China

${ }^{2}$ Tianjin Lung Cancer Center, Tianjin, China

Full list of author information is available at the end of the article
}

the underlying mechanism remains unclear. Therefore, the study of signaling pathways related to erlotinib-induced radiosensitization, understanding the survival mechanisms of tumor cells under the combined treatment with erlotinib and radiation, and further exploration of methods to enhance the radiosensitizing effect of erlotinib may have potential clinical significance.

\section{Materials and method}

Reagents and cell culture

RPMI-1640 culture medium was obtained from Gibco (USA, Grand Island), and fetal bovine serum was obtained from Hangzhou Sijiqing Biological Engineering Materials Co., Ltd. Monoclonal antibodies targeting c-MET, 
phosphorylated c-MET, PI3K, AKT, and phosphorylated AKT were purchased from Santa Cruz Biotechnology, Inc. (USA, Dallas, Texas). RNase A and propidium iodide (PI) were obtained from Sigma (USA, St. Louis, MO). The $\mathrm{CO}_{2}$ incubator used for cell culture was purchased from Heraeus (Germany, Frankfurt), and the high-speed refrigerated centrifuge was also obtained from Heraeus. The flow cytometer was from Beckman Coulter, Inc. (USA, California). The A973 lung adenocarcinoma cell line was used in this study. A973 cells expressed high levels of epidermal growth factor receptor (EGFR) and phospho-EGFR, as reported previously [5-7]. Cells were cultured in RPMI-1640 medium supplemented with $10 \%$ fetal bovine serum, $100 \mathrm{IU} / \mathrm{ml}$ penicillin, and $100 \mathrm{IU} / \mathrm{ml}$ streptomycin in a $37^{\circ} \mathrm{C}$ incubator with an atmosphere of $5 \% \mathrm{CO}_{2}$. Cells in the exponential growth phase were irradiated.

\section{Colony formation assay}

Colony-forming rates of the tumor cells were determined using the colony formation assay. The experiments on erlotinib-induced radiosensitization included the following treatment groups: blank control group, radiation alone group, erlotinib alone group, anti-c-MET monoclonal antibody alone group, combined erlotinib and radiation group, and combined erlotinib and radiation with anti-c-MET monoclonal antibody group. Cells in the exponential growth phase were trypsinized, counted, diluted, and seeded onto 35-ml flasks. The number of cells seeded onto the flasks was adjusted according to the radiation dose $(500,1000,2000,4000,6000,8000$, and 10000 cells were seeded in $0,1,2,4,6,8$, and 10 Gy groups, respectively). The concentrations of erlotinib and anti-c-MET monoclonal antibody used were $20 \mathrm{nM}$. and $10 \mathrm{nM}$, respectively. A radiation dose of $2 \mathrm{~Gy} / \mathrm{min}$ was selected, and cells were exposed to 0 , $1,2,4,6,8$, or 10 Gy of radiation after the attachment of cells on the plastic at $37^{\circ} \mathrm{C}, 5 \% \mathrm{CO}_{2}$, and constant humidity. After 14 days of cell seeding, the culture dishes were collected, and the culture medium was discarded. Cells were fixed and subjected to Giemsa staining. The number of colonies containing more than 50 cells was counted, and the cell survival fraction (SF) was calculated. The experiments were confirmed three times, and each treatment group contained three parallel samples. The single-hit, multitarget model was used to fit the cell survival curves [8-10].

\section{Flow cytometry}

Cell apoptosis and the expression of c-MET were examined by flow cytometry. Experiments included the following treatment groups: blank control group, radiation alone group, erlotinib alone group, and combined erlotinib and radiation group. The concentrations of erlotinib and the anti-c-MET monoclonal antibody were the same as those listed in the preceding section. All irradiated groups were given a dose of 6 Gy. Colony-forming cells from different treatment groups were collected, and the apoptotic rates and expression of c-MET were determined by flow cytometry. Apoptosis was measured experimentally as follows: first, cells were trypsinized, and $5 \times 10^{5}$ cells were collected. After adding $1 \mathrm{ml}$ of cold phosphate buffered saline (PBS), the cells were centrifuged at $1000 \mathrm{rpm}$ for $10 \mathrm{~min}$ at $4^{\circ} \mathrm{C}$. The cells were then washed with PBS, centrifuged twice under the above conditions, and resuspended in $200 \mu \mathrm{l}$ of binding buffer. Ten microliters of Annexin-FITC was added to the cell suspension and mixed well. The cell mixture was incubated at room temperature in the dark for $15 \mathrm{~min}$, and then an additional $300 \mu \mathrm{l}$ of binding buffer was added. Finally, the cells were analyzed by flow cytometry after adding $5 \mu \mathrm{l}$ of PI. To detect the expression of c-MET, the cells were incubated with the PE-coupled mouse anti-human c-MET monoclonal antibody, washed, incubated with the FITC-coupled rabbit anti-mouse antibody, washed, and then subjected to flow cytometry analysis $[11,12]$.

\section{Western blotting}

The expressions of c-MET, p-c-MET, PI3K, AKT, and $\mathrm{p}$-AKT in the blank control group, the combined erlotinib and radiation group, and the combined erlotinib and radiation with anti-c-MET monoclonal antibody group were examined using Western blotting,. The concentrations of erlotinib and the anti-c-MET monoclonal antibody were the same as those described above. Cells were irradiated at a dose of 6 Gy. The experimental procedures were performed as follows: 14 days after treatment, the cells were trypsinized and collected. The total protein was extracted from the cells, and the protein concentration was determined by Coomassie brilliant blue staining. The proteins were separated by polyacrylamide gel electrophoresis and transferred onto polyvinylidene difluoride membranes. The membranes were then probed with primary antibodies, washed, incubated with horseradish peroxidase-conjugated secondary antibodies, and washed again. Finally, protein signals were visualized [13,14].

\section{Statistical analysis}

Origin7.5 software was used to fit the cell survival curves. Data were presented as the mean \pm standard deviation and were analyzed using SPSS17.0 software. The analysis of variance (ANOVA) tables were used to perform comparisons between multiple groups. P values less than 0.05 were considered statistically significant.

\section{Patient consent}

The study was not related with patients. 


\section{Results}

Apoptosis and colony formation under the combined treatment of erlotinib and $6 \mathrm{~Gy}$ of radiation

Our results showed that the apoptotic rates of cells in the blank control group, erlotinib alone group, radiation alone group, and combined erlotinib and radiation group after 24 hours of treatment were $2.43 \pm 1.03 \%, 11.26 \pm$ $2.14 \%, 23.45 \pm 4.35 \%$, and $47.68 \pm 6.73 \%$, respectively. The apoptotic rate in the combined treatment group was significantly higher than those in the other groups (ANOVA analysis, $\mathrm{P}<0.05$ ). The number of colonies was counted after 14 days of treatment, and the colonyforming rates of cells in the blank control group, erlotinib alone group, radiation alone group, and combined erlotinib and radiation group were $71.45 \pm 4.64 \%$, $43.56 \pm 3.38 \%$, $15.6 \pm 2.26 \%$, and $2.04 \pm 1.02 \%$, respectively. Although the colony-forming rate in the combined treatment group was the lowest (Figure 1, ANOVA analysis, $\mathrm{P}<0.05$ ). These results showed that erlotinib exhibited a clear radiosensitizing effect. However, a portion of the tumor cells survived the combined treatment with erlotinib and radiation and eventually formed colonies.

The expression of c-MET in colony-forming cells after the combined treatment with erlotinib and radiation

Cells from the blank control group, the erlotinib alone group, the radiation ( $6 \mathrm{~Gy}$ ) alone group, and the combined erlotinib and radiation group after 14 days of treatment were collected, and the results of the flow cytometry analysis showed that the expression rates of c-MET in these groups were $12.25 \pm 2.17 \%, 23.36 \pm 3.16 \%, 27.45 \pm 3.79 \%$, and $75.35 \pm 6.33 \%$, respectively. Compared to other groups, the expression rate of $\mathrm{c}$-MET in the combined treatment group significantly increased (Figure 2, ANOVA analysis, $\mathrm{P}<0.05)$. These results showed that both radiation and erlotinib were contributing factors to the increased c-MET expression. Moreover, the combination of radiation and erlotinib had a synergistic effect on the upregulation of c-MET expression.

\section{The inhibition of c-MET activity further enhances the radiosensitizing effect of erlotinib}

The survival curves showed that erlotinib exhibited a clear radiosensitizing effect. Compared to the combined erlotinib and radiation group, the inhibition of the c-MET signaling pathway in these cells using a monoclonal antibody further decreased the SF and enhanced the radiosensitizing effect (ANOVA analysis, $\mathrm{P}<0.05$, Figure 3 ). The $\mathrm{D}_{0}, \mathrm{~N}$, and $\mathrm{Dq}$ values for the radiation alone group were $2.51,2.93$, and 1.17, respectively, and for the combined erlotinib and radiation group were $1.54,1.62$, and 0.32 , respectively; the sensitizer enhancement ratio was 1.63. The $D_{0}, N$, and Dq values for the combined erlotinib and radiation with anti-c-MET monoclonal antibody treatment group were $1.01,1.17$, and 0.07 , respectively, and the sensitizer enhancement ratio was 2.49. Therefore, the elevated c-MET expression likely acted as a survival factor for the tumor cells treated with a combination of erlotinib and radiation, and the blockade of c-MET signaling significantly enhanced the tumor cytotoxicity effects.

\section{Changes in protein expression related to the c-MET-PI3K-AKT pathway in colony-forming cells from the combined erlotinib and radiation group before and after c-MET inhibition}

To study the possible survival mechanism utilized by colony-forming cells, cells from the blank control group, the combined erlotinib and radiation group, and the combined erlotinib and radiation with anti-c-MET monoclonal antibody group were collected after 14 days of treatment, and the expression of c-MET, phosphorylated c-MET, PI3K, AKT, and phosphorylated AKT was examined. The results showed that the expression of these proteins significantly increased after 14 days of treatment.

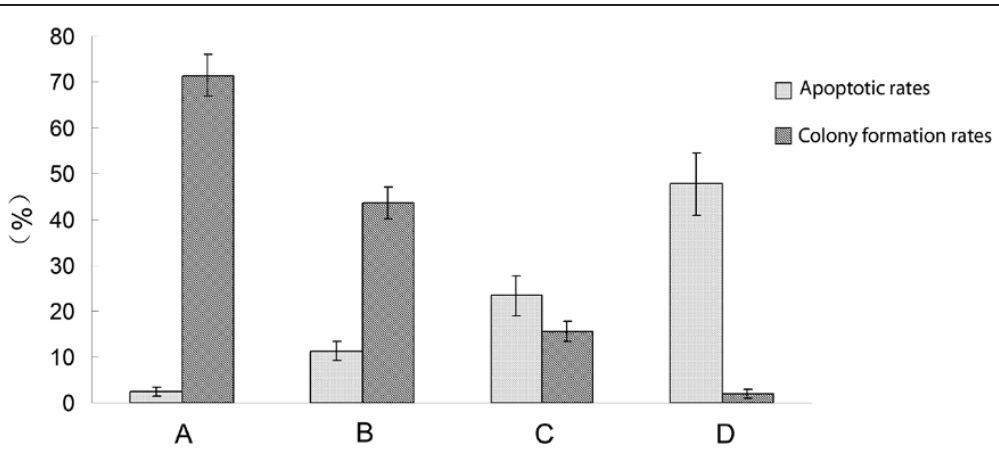

Figure 1 Apoptosis and colony formation under the combined treatment of erlotinib(20 nM) and 6 Gy radiation. (A) Control group: the apoptotic rate was $2.43 \pm 1.03 \%$; the colony formation rate was $71.45 \pm 4.64 \%$. (B) Erlotinib group: the apoptotic rate was $11.26 \pm 2.14 \%$; the colony formation rate was $43.56 \pm 3.38 \%$. (C) Radiation group: the apoptotic rate was $23.45 \pm 4.35 \%$; the colony formation rate was $15.6 \pm 2.26 \%$. (D) combined treatment with erlotinib and radiation group: the apoptotic rate was $47.68 \pm 6.73 \%$; the colony formation rate was $2.04 \pm 1.02 \%$. 

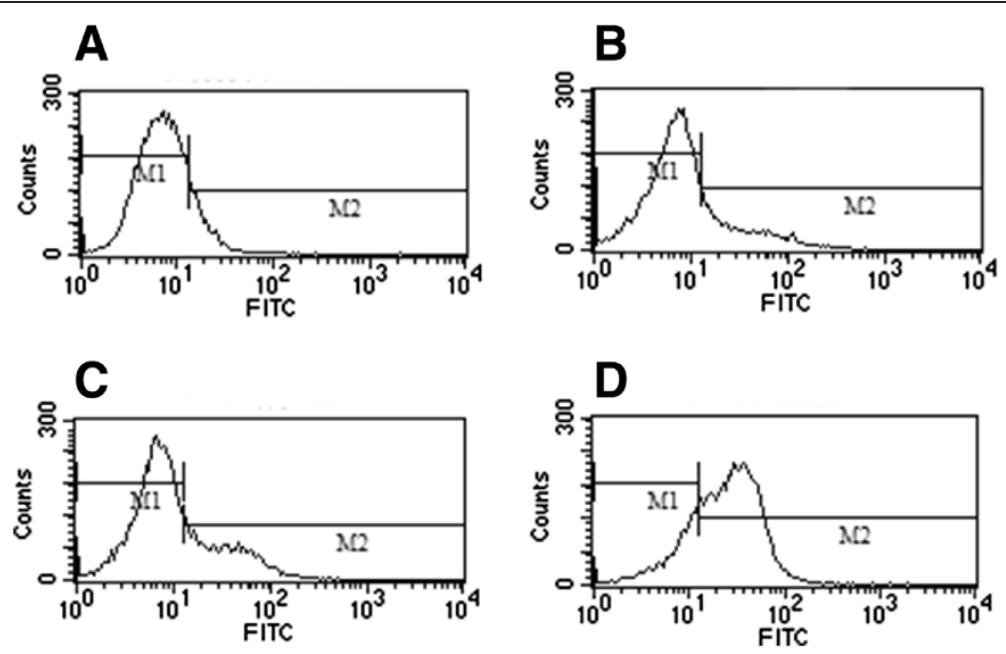

Figure 2 The expression of c-MET in colony-forming cells after combined treatment with erlotinib (20 $\mathrm{nM}$ ) and radiation (6Gy). M1: the cell of c-MET non-expression. M2: the cell of c-MET expression. The expressions of c-MET (M2) were as follows: (A) Control group: $12.25 \pm 2.17 \%$. (B) Erlotinib group: $23.36 \pm 3.16 \%$. (C) Radiation group: $27.45 \pm 3.79 \%$. (D) combined treatment with erlotinib and radiation group: $75.35 \pm 6.33 \%$.

However, after treatment with the anti-c-MET monoclonal antibody, the expression of these proteins significantly decreased (Figure 4). Moreover, the changes in protein expression observed before and after inhibiting the c-MET-PI3K-AKT pathway using the anti-c-MET monoclonal antibody further indicated that the activation of the c-MET-PI3K-AKT pathway was an important mechanism for A973 cell survival and colony formation after the combined treatment with erlotinib and radiation. Therefore, blockade of this pathway may represent a novel

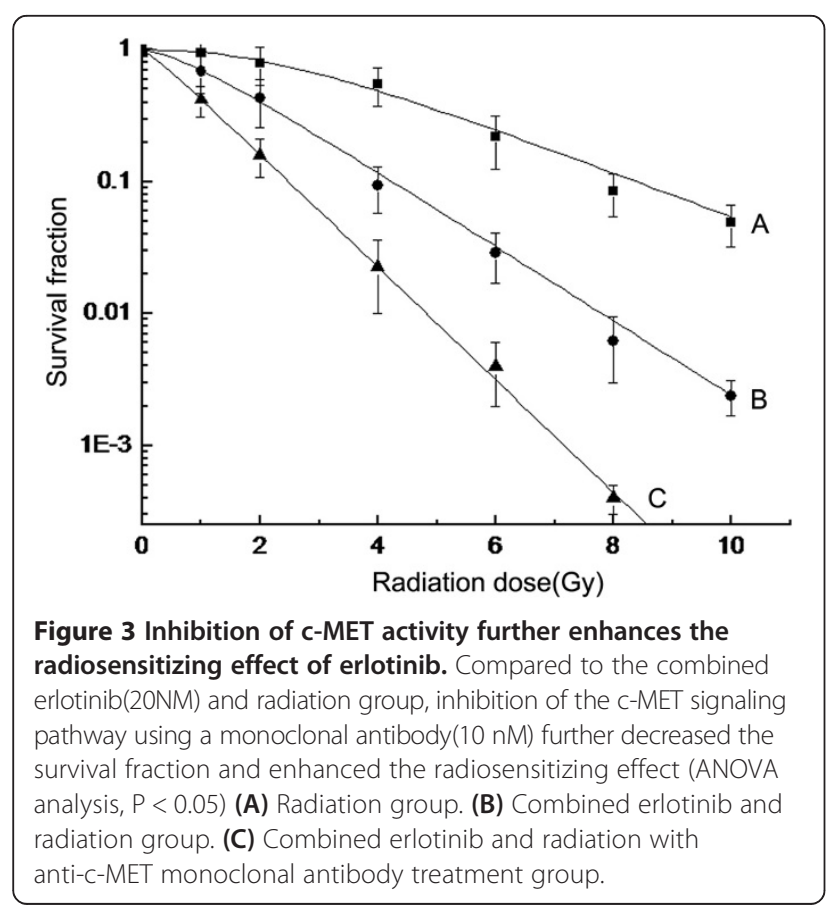

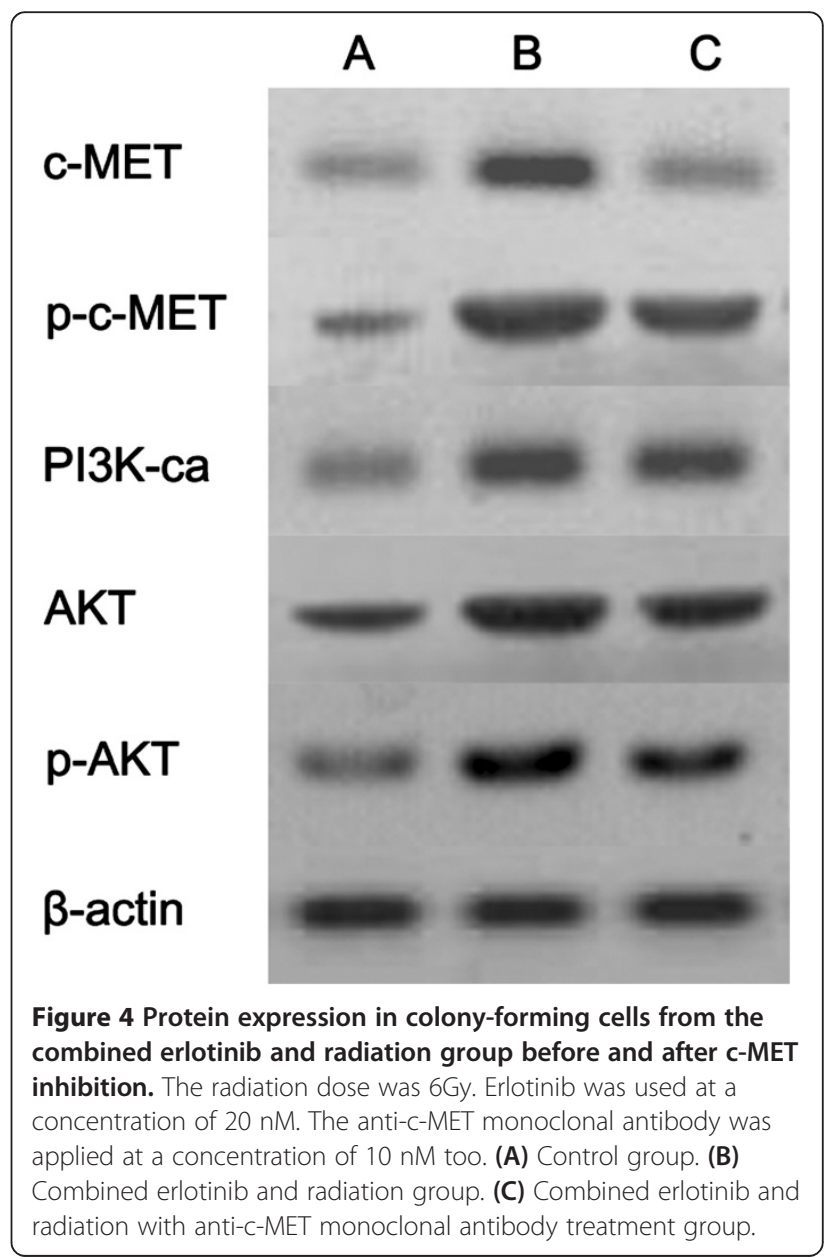


approach for enhancing the radiosensitizing effect of erlotinib.

\section{Discussion}

The results of the present study indicated that the activation of the c-MET-PI3K-AKT pathway served as an important survival mechanism for cells that received the combined treatment with erlotinib and radiation. However, blockade of the c-MET-PI3K-AKT pathway could further enhance the radiosensitizing effect of erlotinib.

Both erlotinib and radiation play important roles in activating the c-MET-PI3K-AKT pathway, which is one of the main causes of acquired resistance to erlotinib. After erlotinib treatment, the PI3K signaling pathway is significantly upregulated in tumor cells. Consequently, downstream pathways and nuclear gene expression are activated, resulting in tumor cell survival and proliferation $[15,16]$. In addition, many studies have shown that radiation stimulates the PI3K pathway [17-20], leading to radiation resistance in tumor cells. Under the combined action of erlotinib and radiation, the likelihood of c-MET-PI3K-AKT pathway activation significantly increases. Based on these findings, we selected c-METPI3K-AKT pathway to evaluate in our research. The mechanism by which the activated c-MET-PI3K-AKT pathway promotes tumor cell survival involves cross-talk between cell signaling pathways [21-24]. Normally, the EGFR/MAPK (mitogen-activated protein kinase) pathway is one of the major pathways driving tumor cell proliferation, whereas the activity of the PI3K pathway decreases due to the function of phosphatase and tensin homolog (PTEN). Upon erlotinib treatment, the MAPK pathway is inhibited and the expression of PTEN is downregulated, resulting in the decreased inhibition of the PI3K pathway $[25,26]$. Owing to the cross-communication between these pathways, the PI3K pathway is stimulated. Similarly, radiation further enhances the activation of the PI3K pathway. As a result, the activity of the PI3K pathway increases significantly, which maintains cell survival and proliferation $[27,28]$.

The results of the present study support those of previous studies mentioned in the preceding paragraph, because in the present study erlotinib exhibited a clear radiosensitizing effect. However, many cells survived under the combined effect of radiation and erlotinib. The survival of such cells may be because both radiation and erlotinib can promote the expression of c-MET, which subsequently activates downstream pathways and affects their cytotoxic effects. Our finding that the inhibition of c-MET with a monoclonal antibody decreased the SF of tumor cells and downregulated the c-MET-PI3K-AKT pathway activity further confirmed the mechanism of tumor cell survival, and these results may provide a new approach to further enhance the cytotoxic effects of combined therapy with erlotinib and radiation in tumor cells (Figure 3).

Previous studies have mainly focused on the molecular mechanism of erlotinib-induced radiosensitization and cytotoxicity [2,3,29], and few have studied the survival mechanism of tumor cells given the combined treatment with erlotinib and radiation. Furthermore, studies exploring the connection between erlotinib-induced radiosensitization and the related signaling pathways are even rarer [30,31]. However, our present study sought to address these shortcomings, and we evaluated the c-MET-PI3KAKT pathway to study protein expression changes following the combined treatment with radiation and erlotinib, as well as the regulatory effects of blocking of this pathway on erlotinib-induced radiosensitization. Furthermore, the current study preliminarily explored the survival mechanism of tumor cells receiving the combined treatment with erlotinib and radiation and provided constructive ideas for how to enhance the radiosensitizing effect of erlotinib.

\section{Current and future developments}

In summary, this study performed constructive investigations of changes in the activity of the c-MET-PI3K-AKT pathway under the combined treatment with erlotinib and radiation and the regulatory effects of blocking the c-MET-PI3K-AKT pathway on the radiosensitizing effect of erlotinib. Our findings revealed an important mechanism for cell survival under the combined treatment with erlotinib and radiation and provided a new experimental basis for further enhancing the radiosensitizing effect of erlotinib. Although only in vitro experiments were performed in this study and many questions remained to be fully explored, we believe that with further in-depth basic research, erlotinib-induced radiosensitization will achieve superior efficacy and possess a broad clinical application.

\section{Abbreviations \\ EGFR: Epidermal growth factor receptor; MET: Mesenchymal-epithelial transition factor; PI3K: PI 3-kinases; FITC: Fluorescein isothiocyanate.}

\section{Competing interests}

We declare that the manuscript has not been published or submitted for publication elsewhere and there is no financial support which may pose conflict of interest. We acknowledge that all authors have contributed significantly, and that all authors are in agreement with the content of the manuscript.

\section{Authors' contributions}

$\mathrm{HQZ}, \mathrm{HZ}$, ZLJ and PW participated in the design of the study; $H Q Z, H Z, Q B$, YG performed the research; JW and LZ performed the statistical analysis, $\mathrm{HQZ}$ and $\mathrm{HZ}$ wrote the paper. All authors read and approved the final manuscript.

\section{Acknowledgements}

This research was funded by Tianjin Health Bureau Science and Technology Development Fund (2012KZ066) and National Natural Science Foundation of China (81301925). 


\section{Author details}

${ }^{1}$ Department of Radiotherapy, Tianjin Medical University Cancer Institute and Hospital, National Clinical Research Center for Cancer, Tianjin Key Laboratory of Cancer Prevention and Therapy, Tianjin, China. ${ }^{2}$ Tianjin Lung Cancer Center, Tianjin, China. ${ }^{3}$ Department of Hematology, Weifang People's Hospital, Weifang, Shandong province, China. ${ }^{4}$ Department of Oncology, Affiliated Hospital of Weifang Medical University, Weifang, Shandong province, China.

Received: 1 August 2013 Accepted: 20 October 2014

Published online: 30 November 2014

\section{References}

1. Mehta VK: Radiotherapy and erlotinib combined: review of the preclinical and clinical evidence. Front Oncol 2012, 2:31.

2. Z Zhuang H, Wang J, Zhao L, Yuan Z, Wang P: The theoretical foundation and research progress for WBRT combined with erlotinib for the treatment of multiple brain metastases in patients with lung adenocarcinoma. Int J Cancer 2013, 133(10):2277-2283.

3. Zhuang H, Yuan Z, Wang J, Zhao L, Pang Q, Wang P: Phase II study of whole brain radiotherapy with or without erlotinib in patients with multiple brain metastases from lung adenocarcinoma. Drug Des Devel Ther 2013, 7:1179-1186.

4. Wykosky J, Fenton T, Furnari F, Cavenee WK: Therapeutic targeting of epidermal growth factor receptor in human cancer: successes and limitations. Chin J Cancer 2011, 30(1):5-12.

5. Zhuang $\mathrm{HQ}, \mathrm{Bo} \mathrm{QF}$, Jun W: The different radiosensitivity when combining erlotinib with radiation at different administration schedules might be related to activity variations in C-MET-PI3K-AKT signal transduction. Onco Targets Ther 2013, 6:603-608.

6. Liu F, Li Y, Yu Y, Fu S, Li P: Cloning of novel tumor metastasis-related genes from the highly metastatic human lung adenocarcinoma cell line Anip973. J Genet Genomics 2007, 34:189-195.

7. Xu QY, Gao Y, Liu Y, Yang WZ, Xu XY: Identification of differential gene expression profiles of radioresistant lung cancer cell line established by fractionated ionizing radiation in vitro. Chin Med J (Engl) 2008, 121:1830-1837.

8. Chu PM, Chiou SH, Su TL, Lee YJ, Chen LH, Chen YW, Yen SH, Chen MT, Chen $\mathrm{MH}$, Shih $\mathrm{YH}$, Tu PH, Ma HI: Enhancement of radiosensitivity in human glioblastoma cells by the DNA N-mustard alkylating agent BO-1051 through augmented and sustained DNA damage response. Radiat Oncol 2011, 19;6:7.

9. Harari PM, Chinnaiyan P, Huang SJ: Combined Treatment With Radiation And An Epidermal Growth Factor Receptor. 2009. US 12455750.

10. Quanz M, Berthault N, Roulin C, Roy M, Herbette A, Agrario C, Alberti C, Josserand V, Coll JL, Sastre-Garau X, Cosset JM, Larue L, Sun JS, Dutreix M: Small-molecule drugs mimicking DNA damage: a new strategy for sensitizing tumors to radiotherapy. Clin Cancer Res 2009, 15(4):1308-1316.

11. Combs AP, Sparks RB, Maduskuie TP Jr: Bicyclic Azaheterocyclobenzylamines As Pi3k Inhibitors. 2013. US20130261101A1.

12. Dassonville O, Bozec A, Fischel JL, Milano G: EGFR targeting therapies: monoclonal antibodies versus tyrosine kinase inhibitors Similarities and differences. Crit Rev Oncol Hemato 2007, 62:53-61.

13. Kim IA, Shin JH, Kim IH, Kim JH, Kim JS, Wu HG, Chie EK, Ha SW, Park Cl, Kao GD: Histone deacetylase inhibitor-mediated radiosensitization of human cancer cells: class differences and the potential influence of p53. Clin Cancer Res 2006, 12(3):940-949.

14. Ma PC, Jagadeeswaran $R$, Jagadeesh $S$, Tretiakova MS, Nallasura $V$, Fox EA, Hansen M, Schaefer E, Naoki K, Lader A, Richards W, Sugarbaker D, Husain AN, Christensen JG, Salgia R: Functional expression and mutations of C-Met and its therapeutic inhibition with SU11274 and small interfering RNA in non-small cell lung cancer. Cancer Res 2005, 65(4):1479-1488.

15. Bidkhori G, Moeini A, Masoudi-Nejad A: Modeling of tumor progression in NSCLC and intrinsic resistance to TKI in loss of PTEN expression. PLoS One 2012, 7(10):e48004.

16. Kang XH, Xu ZY, Gong YB, Wang LF, Wang ZQ, Xu L, Cao F, Liao MJ: Bufalin Reverses HGF-Induced Resistance to EGFR-TKIs in EGFR Mutant Lung Cancer Cells via Blockage of Met/PI3k/Akt Pathway and Induction of Apoptosis. Evid Based Complement Alternat Med 2013, 2013:243859.

17. Xu S, Hege KM, Tran TM: Methods For Treating Cancer Using Tor Kinase Inhibitor Combination Therapy. 2013. US20130225518A1.
18. Kuger S, Graus D, Brendtke R, Günther N, Katzer A, Lutyj P, Polat B, Chatterjee M, Sukhorukov VL, Flentje M, Djuzenova CS: Radiosensitization of glioblastoma cell lines by the dual PI3K and mTOR inhibitor NVP-BEZ235 depends on drug-irradiation schedule. Trans/ Oncol 2013, 6(2):169-179.

19. De Bacco F, Luraghi P, Medico E, Reato G, Girolami F, Perera T, Gabriele P, Comoglio PM, Boccaccio C: Induction of MET by ionizing radiation and its role in radioresistance and invasive growth of cancer. J Natl Cancer Inst 2011, 103:645-661.

20. Liu X, Yao W, Newton RC, Scherle PA: Targeting the c-MET signaling pathway for cancer therapy. Expert Opin Investig Drugs 2008, 17:997-1011.

21. Maines MD: Biliverdin reductase: PKC interaction at the cross-talk of MAPK and PI3K signaling pathways. Antioxid Redox Signal 2007, 9:2187-2195.

22. Aksamitiene E, Kiyatkin A, Kholodenko BN: Cross-talk between mitogenic Ras/MAPK and survival PI3K/Akt pathways: a fine balance. Biochem Soc Trans 2012, 40:139-146.

23. Menges CW, McCance DJ: Constitutive activation of the Raf-MAPK pathway causes negative feedback inhibition of Ras-PI3K-AKT and cellular arrest through the EphA2 receptor. Oncogene 2008, 27:2934-2940.

24. Jung KH, Park BH, Hong SS: Progress in cancer therapy targeting c-Met signaling pathway. Arch Pharm Res 2012, 35:595-604.

25. Burris HA 3rd: Overcoming acquired resistance to anticancer therapy: focus on the PI3K/AKT/mTOR pathway. Cancer Chemother Pharmacol 2013, 71:829-842.

26. Park JK, Jung HY, Park SH, Kang SY, Yi MR, Um HD, Hong SH: Combination of PTEN and gamma-ionizing radiation enhances cell death and G(2)/M arrest through regulation of AKT activity and p21 induction in non-small-cell lung cancer cells. Int J Radiat Oncol Biol Phys 2008, 70:1552-1560.

27. Haley JD, Thomson S: Biological Markers Predictive Of Anti-Cancer Response To Kinase Inhibitors. 2013. US20130210026A1.

28. Contessa JN, Hampton J, Lammering G, Mikkelsen RB, Dent P, Valerie K, Schmidt-Ullrich RK: lonizing radiation activates Erb-B receptor dependent Akt and p70 S6 kinase signaling in carcinoma cells. Oncogene 2002, 21:4032-4041.

29. Huang S, Li C, Armstrong EA, Peet CR, Saker J, Amler LC, Sliwkowski MX, Harari PM: Dual targeting of EGFR and HER3 with MEHD7945A overcomes acquired resistance to EGFR inhibitors and radiation. Cancer Res 2013, 73:824-833.

30. Cai SX: Small molecule vascular disrupting agents: potential new drugs for cancer treatment. Recent Pat Anticancer Drug Discov 2007, 2:79-101.

31. Ortiz R, Melguizo C, Prados J, Álvarez PJ, Caba O, Rodríguez-Serrano F, Hita $F$, Aránega A: New gene therapy strategies for cancer treatment: a review of recent patents. Recent Pat Anticancer Drug Discov 2012, 7:297-312.

doi:10.1186/s12935-014-0109-5

Cite this article as: Zhuang et al:: Experimental study on the regulation of erlotinib-induced radiosensitization with an anti-cMET monoclonal antibody. Cancer Cell International 2014 14:109.

\section{Submit your next manuscript to BioMed Central and take full advantage of:}

- Convenient online submission

- Thorough peer review

- No space constraints or color figure charges

- Immediate publication on acceptance

- Inclusion in PubMed, CAS, Scopus and Google Scholar

- Research which is freely available for redistribution 